\title{
Vapor-liquid solid mechanism using gold colloids for the growth of GaAs nanowires
}

\author{
Rosnita Muhammad $^{1, *}$, Zulkafli Othaman ${ }^{1}$, Samsudi Sakrani ${ }^{1}$, Yussof Wahab ${ }^{2}$ \\ ${ }^{1}$ Physics Department, Faculty of Science, Universiti Teknologi Malaysia, 81310 UTM, Skudai, Johor \\ ${ }^{2}$ Ibnu Sina Institute, Universiti Teknologi Malaysia, 81310 UTM, Skudai, Johor \\ * To whom correspondence should be addressed. E-mail: rosnita@utm.my
}

Received: 23 October 2008

http://dx.doi.org/10.11113/mjfas.v4n2.44

\section{ABSTRACT}

GaAs nanowires were grown on the semi insulating undoped GaAs (111)B wafer by using metal organic chemical vapor deposition, MOCVD. The growth follows the vapor-liquid-solid method by applying nanoparticle gold colloid as a catalyst to forms a eutectic liquid alloy with the wafer substrate. The GaAs wafer were first dipped in the poly-L-lysine solution before $30 \mathrm{~nm}$ gold colloid dropped on the wafer surface. The temperature growth was set at $420^{\circ} \mathrm{C}$ and the growth processes were done between 10 to $60 \mathrm{~min}$. The samples structural were characterized using field emmission scanning electron microscope, FE-SEM and shows the GaAs nanowires height increased with the increasing growth time. On the top of each wire contain ball which include some quantity of Au when anlyze using energy disperve x-ray spectroscopy, EDX.

| Vapor-liquid solid mechanism| GaAs | Nanowire | MOCVD |

\section{Introduction}

A nanowire (NW) is an extremely thin wire with a diameter on the order of a few nanometer (nm) or less. Free standing NWs, also called nanowhiskers, have a promising future as one-dimensional (1D) building blocks for a wide range of applications for example in electronics, photonics and life-sciences [1]. NWs have many interesting properties that are not seen in bulk or 3-D materials because electrons in NWs are quantum confined laterally and thus occupy energy levels. NWs can be produced using chemical etching, indent the STM tip near the surface, deposited or synthesize from the elements. A common technique for creating a NW is the vaporliquid-solid (VLS) synthesis method which first discovered by Wagner and Ellis [2] in 1964 to explain the epitaxial growth of micron-sized Si whiskers. According to this method, a small (micrometer-sized) Au particle on the semiconductor Si surface forms a eutectic liquid alloy with the host material at growth temperature. The liquid droplet is an ideal sink for reaction species supplied from the surrounding vapor and is readily supersaturated with reaction species. The precipitation of reaction species predominantly occurs at the liquid/solid interface and leads to highly anisotropic growth. Recently, the VLS growth technique has been extended to nm-sized Au particles on a variety of semiconductor materials including III-V [3,4], II-VI [5] compound and group IV elemental [6] semiconductors. With a reduction in size, unique electrical, mechanical, chemical and optical properties would be results of large surface area and quantum confinement effects [1,7-8]. 
III-V compound semiconductor NWs such as GaAs was intensively being investigated due to the direct band gap and high carrier mobility [9]. However, there are still many open questions to be answered before GaAs whiskers can be used as versatile building blocks in nanostructure devices. In this study, we investigated the growth of GaAs NWs using MOCVD with different growth time processes between 10 to $60 \mathrm{~min}$.

\section{Experimental}

The experiments start with semi insulating GaAs (111)B wafer immersed in $0.1 \%$ poly-L-lysine (PLL) solution for $3 \mathrm{~min}$. After washing with deionize water and subsequent drying with $\mathrm{N}_{2}$, the wafer was cut into desired size. The $30 \mathrm{~nm}$ diameter gold colloids were dispersed on the wafer surface by using microlittre pipette and immediately washed after $20 \mathrm{sec}$. The PLL layer on the surface is positively charged and attracts the negatively charged of the gold colloids [10]. The cutting wafer was put on the graphite susceptor inside the vertical reactor in the MOCVD system for the growth processes. The growth pressure inside vertical reactor chamber was 76 Torr. Trimethylgallium (TMGa) and arsine $\left(\mathrm{AsH}_{3} ; 10 \%\right.$ in $\left.\mathrm{H}_{2}\right)$ were used as the source gases. The $\mathrm{AsH}_{3}$ to TMGa molar flow ratio (the V/III ratio) was set at 80 . The wafer was annealed in situ at $600^{\circ} \mathrm{C}$ under $\mathrm{AsH}_{3}$ ambient for $10 \mathrm{~min}$ to desorbed surface contaminants and form eutectic alloy between Ga and gold colloid (Au) [3]. All the samples were characterized using a JEOL JSM-6701F field emission scanning electron microscope (FE-SEM) system. It operated at $2 \mathrm{kV}$ for tilted views picture to obtain information on surface density (number of nanowires perunit area) and morphology (growth orientation on the substrate, height and faceting orientation). Energy dispersive $\mathrm{x}$-ray spectroscopy (EDX) was used to identify the component elements of the nanowires

\section{Results and Discussions}

Figure 1 shows field emission scanning electron microscopy (FE-SEM) images of GaAs nanowires grown at $420^{\circ} \mathrm{C}$ with different growth time $(10,20,30$ and $60 \mathrm{~min})$ on GaAs (111)B substrate. All the samples were tilted 30 degrees from the electron beam for clarity. Picture (a) shows no nanowire grown except several particles gold colloids with varied sizes are randomly distributed on the GaAs surface. Increasing growth time to 20 min make nanowires built with height up to $97.5 \mathrm{~nm}$. Increasing more to $30 \mathrm{~min}$ growth makes nanowires grown longer. With growth time $60 \mathrm{~min}$, the average wire height is $210 \mathrm{~nm}$ but the densities decreased compare to $30 \mathrm{~min}$ growth. After the nanowire growth process, the samples were cooled in an $\mathrm{AsH}_{3}$ flow to prevent the components of GaAs from desorbing. Most of the nanowires were grown perpendicular to the substrate surface, along the (111)As dangling bond direction. The average height of GaAs nanowires with different growth time process are shown in Figure 2.

The growth-time dependence of the wire growth process indicates that the most likely mechanism for the nanowire growth is vapor-liquid-solid (VLS) mechanisms. This procedure is similar to the ones reported by Hiruma [3] who grown GaAs nanowires using MOCVD system. Au which first deposited on GaAs surface was heated for $10 \mathrm{~min}$ at $600^{\circ} \mathrm{C}$ to desorb surface contaminants. The high temperature $\left(600^{\circ} \mathrm{C}\right)$ is above the Au-Ga binary system eutectic point $\left(353^{\circ} \mathrm{C}\right)[10]$ to promote $\mathrm{Au} / \mathrm{Ga}$ alloy formation. The nanowire/whisker was then grown underneath the droplet of $\mathrm{Au}$ particle when trimethylgallium and $\mathrm{AsH}_{3}$ are supplied. Figure 3 shows a schematic diagram of VLS growth mechanism for GaAs nanowires.

The growth-time dependence of the GaAs nanowire growth is consistent with VLS growth shown in Figure 3. On the top of each wire, there is a characteristic ball. Energy dispersive X-ray spectroscopy (EDX) was used to identify the component elements of the NWs. Several materials were detected as can be seen in Figure 4. 
Spectrum (a) shows the EDX analysis recorded at the point (b) in Figure 2 for GaAs NWs where as spectrum (b) recorded on top of nanowire at point (a). Au peak contains only at point (a). For both spectrums, elements $\mathrm{C}$ probably comes from the TMGa, $\mathrm{O}$ just from exposure to the air because the moment it took out from the reactor, it is exposed to atmosphere. Pt is detected due to the sample coated before EDX process. It is found that from the spectrums, a large quantity of $\mathrm{Ga}$ and As were observed nearly $80 \%$ of the materials contain on each of nanowires. The $\mathrm{Ga}$ and As peaks also reached the highest peak compare to others materials. Nanowire has very large surface area; therefore the Au peak is very small compare to Ga and As.
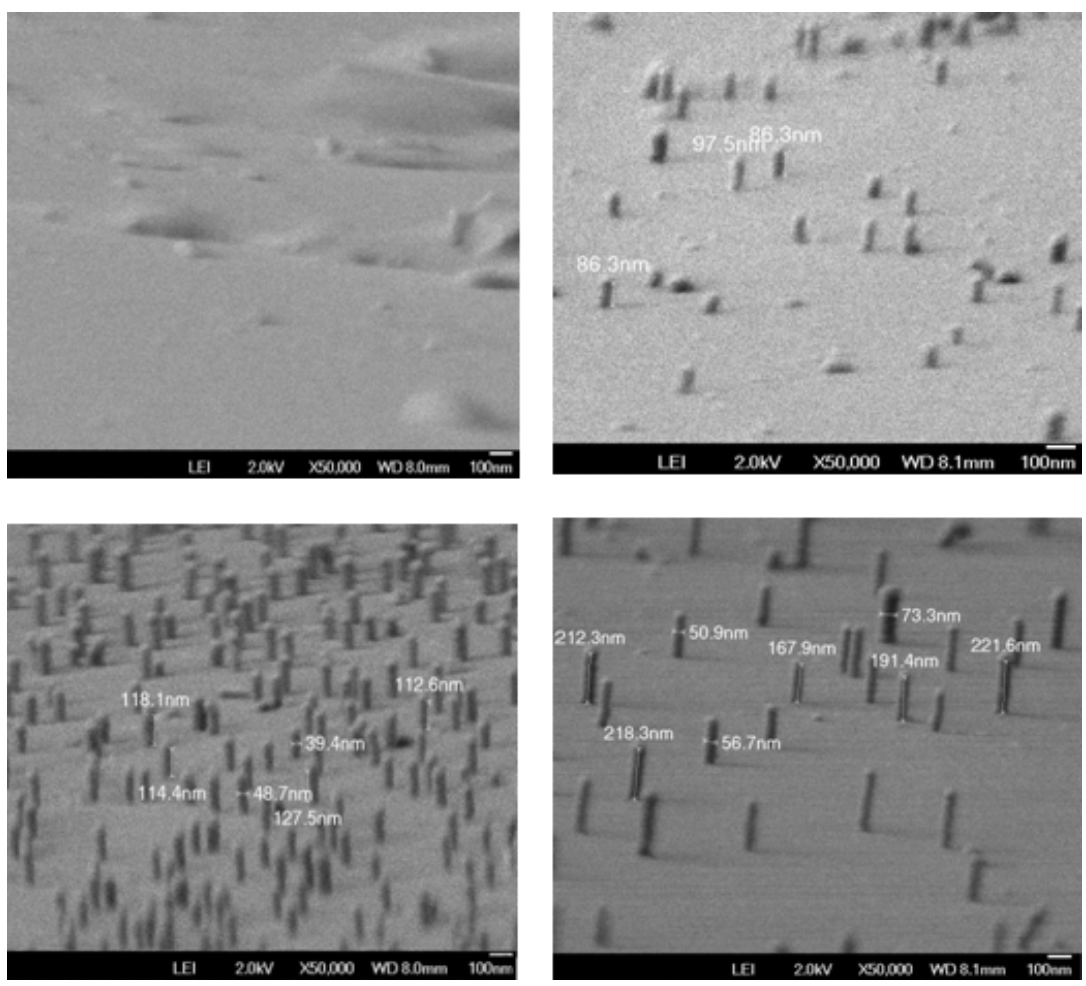

Figure 1: $30^{\circ}$ tilted view of GaAs nanowires grown on a GaAs(111)B substrate for $10 \mathrm{~min}(\mathrm{a}), 20 \mathrm{~min}(\mathrm{~b})$, $30 \mathrm{~min}(\mathrm{c})$, and $60 \mathrm{~min}(\mathrm{~d})$ at a temperature $420^{\circ} \mathrm{C}$ and $\mathrm{V} / \mathrm{III}$ ratio of 80 .

\section{Conclussion}

GaAs nanowires have been prepared on GaAs(111)B substrates by vapor liquid solid mechanisms using metal-organic chemical vapor deposition (MOCVD) system. The microstructural analysis using field-emission scanning electron microscopy system (FE-SEM) shows that most of the nanowires were grown perpendicular to the substrate and the height of nanowires increasing with the growth time increased. On top of each nanowire, a gold ball which plays as a catalyst in forming nanowire was defined by energy dispersive x-ray spectroscopy (EDX). More work has to be done for making nanowires longer and larger surface area for better properties in structural, optical \& electrical properties for electronic and optoelectronic applications 


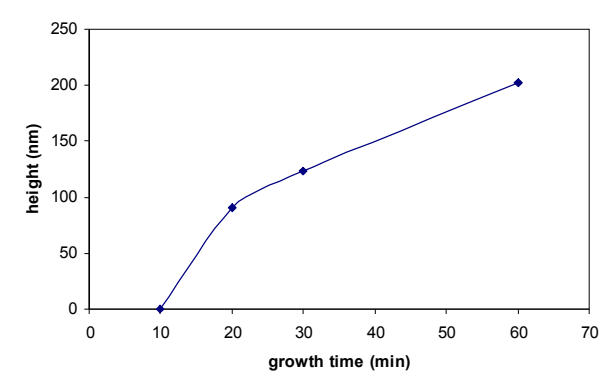

Figure 2: A plot of the nanowire height versus growth time at $420^{\circ} \mathrm{C}$ growth temperature

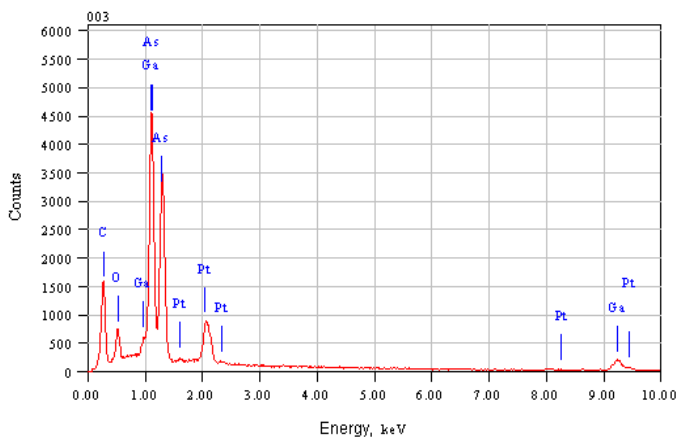

(a) Spectrum recorded at point (b) in Figure 3

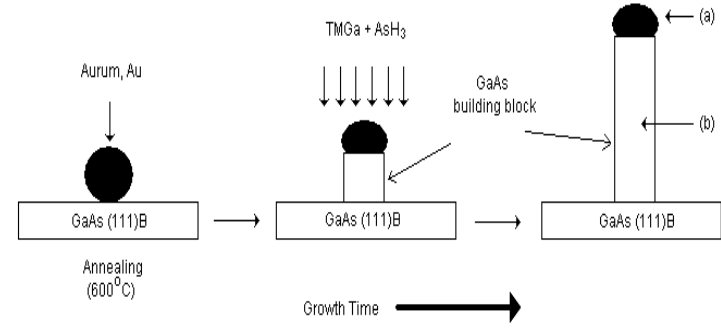

Figure 3: Schematic diagram of vapor-liquid-solid growth of a GaAs nanowire/building block [3]

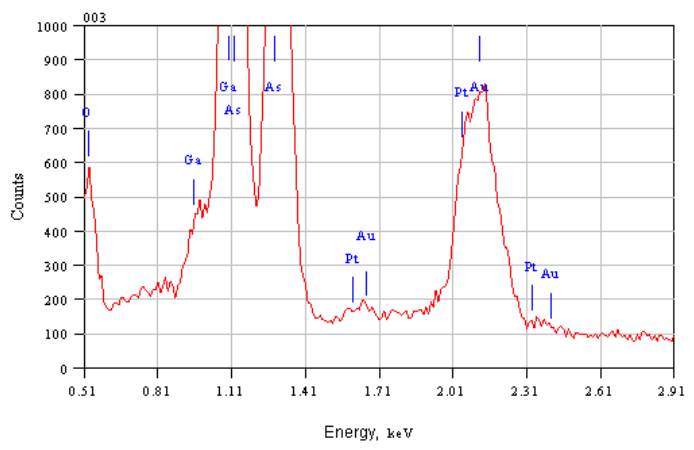

(b) Spectrum recorded at point (a) in Figure 3

Figure 4: EDX spectrum recorded at different positions of the GaAs nanowires. Picture (a) is the spectrum recorded of the GaAs nanowire body. Picture (b) recorded at the tip or head of nanowire.

\section{Acknowledgement}

The authors acknowledge the Ministry of Science, Technology and Environment Malaysia for the financial support through IRPA funding 09-02-06-0056-SR0013/06-01 and also to the Ibnu Sina Institute for Fundamental Science for the laboratory facilities

\section{References}

[1] Lars Samuelson, 2003, "Self-forming nanoscale devices", Materials today, p22.

[2] R. S. Wagner and W. C. Ellis, 1964, "Vapor-liquid solid mechanism of single crystal growth" Applied Physics Letter, Vol 4, No 5, p 89

[3] K. Hiruma, M. Yazawa, T. Katsuyama, K. Ogawa, K. Haraguchi, M. Koguchi and H. Kakibayashi, 1995, "Growth and optical properties of nanometer-scale GaAs and InAs whiskers", Journal of Applied Physics, Vol 77 (2), p 447. 
[4] M. C. Plante and R. R. LaPierre, 2008, "Au-assisted growth of GaAs nanowires by gas source molecular beam epitaxy : Tapering, sidewall faceting and crystal structure", Journal of Crystal Growth, Vol 310, p 356-363.

[5] Q. X. Zhao, M. Willander, R. E. Morjan, Q-H. Hu and E.E. B. Campbell, 2003, "Optical recombination of ZnO nanowires grown on sapphire and Si substrates", Applied Physics Letter, Vol 83, no 1, p 165.

[6] Yi Cui, Lincoln J. Lauhom, Mark S. Gudiksen, Jianfang Wang and Charles M. Lieber, 2001, Diametercontrolled synthesis of single-crystal silicon nanowires", Applied Physics Letter, 78, no 15, p 2214.

[7] M. T. Bjork, B. J. Ohlsson, C. Thelander, A. I. Persson, K. Deppert, L. R. Wallenberg, L. Samuelson, 2002, Applied Physics Letter, 81, p 4458.

[8] L. J. Lauhon, Mark S. Gudiksen and Charles M. Lieber, 2004, "Semiconductor nanowire heterostructure", Phil. Trans. R. Soc. Lond, A, 362, p 1247-1260.

[9] M. R. Brozel and G. E. Stillman, 1996, Properties of Gallium Arsenide, $3^{\text {rd }}$ edition, Inspec, London, UK.

[10] H. H. Tan, K. Sears, S. Mokkapati, Lan Fu, Yong Kim, P. McGowan, M Buda and C. Jagadish, 2006, "QD and Nanowires grown by MOCVD for optoelectronic device applications", IEEE Journal of selected topics in quantum electronics, Vol 12, No 6, p 1242. 